\title{
Influencia de la familia y de los factores sociales en el curso de la enfermedad psiquiátrica.
}

\section{Una comparación entre pacientes esquizofrénicos y depresivos neuróticos ${ }^{1}$.}

\author{
C. E. Vaughn y J. P. Leff
}

\section{INTRODUCCION}

Aunque la etiología de la esquizofrenia sigue preocupando a clínicos e investigadores, actualmente se sabe lo suficiente sobre los precipitantes de recaída de pacientes con trastornos esquizofrénicos claramente establecidos como para sugerir que son personas altamente sensibles a su entorno social. Particularmente interesante es una serie de estudios realizados por George Brown y sus colegas acerca de la influencia de la vida familiar en el curso de los trastornos esquizofrénicos. En un estudio más reciente (Brown, Birley y Wing, 1972) se siguió un método estandarizado para valorar la calidad de la relación emocional entre un paciente esquizofrénico y el familiar con quien convive. Los autores encontraron que podían predecir una recaída de esquizofrenia, durante los nueve meses siguientes al alta, empleando un índice de Emoción Expresada (EE) mostrado por el familiar en una entrevista, poco después del ingreso del paciente en el hospital. Este índice de EE tenía tres componentes, siendo el más importante de ellos el número de comentarios críticos hechos por el familiar al hablar acerca del paciente y de su enfermedad. Otras medidas de la EE eran: hostilidad (que aparecía raramente en ausencia de un elevado número de críticas) y una implicación emocional excesiva ${ }^{2}$.

El índice se urilizó para categorizar pacientes procedentes de familias con EE elevada o baja. Durante los nueve meses siguientes al alta del hospital, el 58 por 100 de pacientes procedentes de hogares con EE elevada recayeron, frente a sólo el 16 por 100 del grupo de EE baja, hallazgo altamente significativo $(p<0,001)$. Esta asociación era independiente tanto del trastorno previo del paciente como de su deterioro laboral.

Los resultados sugerían que en los pacientes que convivían con familiares que habían mostrado una emoción elevada en el momento de la admisión era menos probable la recaída, tanto si habían sido regularmente medicados con fenotiacinas (esta tendencia no alcanzó significación estadística) como si habían podido evitar un contacto directo con la familia. El índice de la EE de los familiares siguió siendo el mejor predictor aislado de recaída sintomática.

La naturaleza inequívoca de estos resultados y sus implicaciones prácticas y teóricas hacían altamente deseable una réplica. Los autores de este 
trabajo, aun compartiendo la misma tradición investigadora de Brown y sus colegas, nos decidimos a llevar a efecto una aproximación a este trabajo desde un punto de vista crítico. Nos interesaba averiguar si los resultados de los factores que influían en la recuperación podían ser replicados con otra muestra de pacientes esquizofrénicos y si los factores eran, de alguna manera, específicos de la esquizofrenia. Decidimos, a efectos comparativos, seleccionar una muestra de pacientes depresivos neuróticos hospitalizados. La muestra de depresivos se limitó a sujetos sin delirios o alucinaciones, con el fin de asegurarnos de que no existía solapamiento diagnóstico alguno con los pacientes esquizofrénicos.

En este informe se da el mayor énfasis a los datos para esquizofréni- cos, si bien se presenta una selección de resultados para pacientes depresivos con objeto de demostrar la importancia relativa de algunas variables en modelos de recaída aplicables a los dos grupos clínicos. En otra parte, presentaremos un análisis más comprensivo de las pautas de recaída en los pacientes depresivos.

El presente estudio es, en muchos aspectos, idéntico al anterior en su diseño y ejecución. Se ha urilizado un diseño prospectivo de seguimiento similar, de nueve meses, con valoración independiente de la conducta pasada, de la respuesta emocional actual de los familiares y de las recaídas subsiguientes. Al igual que en el estudio anterior, la hipótesis a comprobar es «que un grado elevado de emoción expresada es un índice de las características de los familiares que, probablemente, causará una recaída de síntomas floridos, independientemente de otros factores, como duración de la historia clínica, tipo de síntomas o severidad de los trastornos previos de conducta». Y, nuevamente, partiendo de dos supuestos básicos: primero, que el índice de emoción expresada de los familiares es un indicador razonablemente fiable de las relaciones familiares, aunque la conducta habitual, en relación con el paciente, no sea observada directamente; segundo, que la actitud mostrada por los familiares hacia el paciente durante la entrevista es representativa de la forma cotidiana de relacionarse con él. Los hallazgos del estudio de 1972 de Brown et al, parecen justificar estas suposiciones.

\section{DISEÑO}

Los pacientes incluidos en ẹl estudio se seleccionaron secuencialmente en el momento de su recaída, al ser ingresados en uno de los tres hospitales-del sudeste de Londres (Bethlem, Maudsley y St. Francis). Se seleccionaron los historiales de todos los pacientes entre 17 y 64 años cuya lengua nativa era el inglés y que vivían con sus familiares en el momento de su ingreso. Se excluyó a las personas que presentaban indicios de trastornos orgánicos. El psiquiatra J. L. entrevistó a todos los pacientes cuyos historiales sugerían un diagnóstico de esquizofrenia o depresión neurótica, urilizando la novena edición del Examen del Estado Actual (Present State Examination; en adelante PSE) para formular las valoraciones clínicas (Wing, Cooper y Sartorius, 1974). Si se confirmaba el diagnóstico, el paciente era incluido en el estudio y los familiares eran entrevistados por el psicólogo C. V.

Siguiendo estos criterios se seleccionaron, inicialmente, 43 pacientes esquizofrénicos y 32 depresivos. De este número original hubo que excluir a 5 esquizofrénicos y a un depresivo por haber rehusado participar sus familiares ${ }^{3}$. Se eliminaron del estudio dos pacientes más durante el período de nueve meses de seguimiento: uno del grupo depresivo murió durante 
este tiempo y un paciente esquizofrénico dejó su casa al ser dado de alta del hospital. Los restantes pacientes (37 esquizofrénicos y 30 depresivos) constituyeron los grupos finales de seguimiento y representaban, respectivamente, el 86 por 100 y el 94 por 100 de la muestra original. A los pacientes se les distribuyó por sexos y el grupo familiar en que vivían, como se expresa en la tabla 1.

TABLA 1

Distribución de pacientes por diagnóstico, sexo y grupo familiar

\begin{tabular}{|c|c|c|c|c|c|c|}
\hline \multirow[b]{2}{*}{$\begin{array}{l}\text { Familiares con } \\
\text { quienes convive }\end{array}$} & \multicolumn{3}{|c|}{ ESQUIZOFRENICOS } & \multicolumn{3}{|c|}{ DEPRESIVOS } \\
\hline & Hombr & ujeres & Total & Hombres & Iujeres & Total \\
\hline \multirow{3}{*}{$\begin{array}{l}\text { Padres } \\
\text { Cónyuge } \\
\text { Otros (con hijo adulta u otro } \\
\text { familiar }\end{array}$} & 11 & 6 & 17 & 0 & 1. & 1 \\
\hline & 4 & 9 & 13 & 9 & 19 & 28 \\
\hline & 0 & 7 & 7 & 1 & 0 & 1 \\
\hline TOTAL N & 15 & 22 & 37 & 10 & 20 & 30 \\
\hline
\end{tabular}

El promedio de edad de los esquizofrénicos y depresivos era de 33,1 y 38,6 años, respectivamente, diferencia no significativa. Sin embargo, cuando se consideró separadamente a los pacientes varones, los esquizofrénicos eran significativamente más jóvenes (edad al ingreso: esquizofrénicos varones, $x=28,1$ años; depresivos varones, $x=44,8$ años; $t-3,48,23$ g. 1 ., $\mathrm{P}<0,01)$.

Un número elevado de pacientes depresivos vivían con sus esposas, por lo que no fue posible establecer comparaciones intergrupo entre los padres respectivos en las diferentes medidas. La explicación de este hallazgo es necesariamente tentativa; pero podemos pensar que es improbable que, alcanzada cierta edad, un adulto con trastornos depresivos viva con sus padres. En el supuesto de ser soltero, es probable que viva en su casa o con amigos, circunstancia que, desde luego, le excluiría de este estudio. Por otra parte es bien sabido que el porcentaje de casados es bajo entre las personas diagnosticadas de esquizofrenia y que los solteros de este grupo tienden a quedarse en la casa paterna.

Al primer paciente se le vio en octubre de 1971 y la última entrevista de seguimiento tuvo lugar en enero de 1975.

Las entrevistas para el estado mental actual.(PSE) las realizó un psiquiatra poco después de ingresar el paciente en el hospital, repitiéndose al ser reingresado o al seguimiento de nueve meses. El psicólogo administró una versión abreviada de la entrevista familiar (Brown y Rutter, 1966; Rutter y Brown, 1966), en visita domiciliaria, a los pocos días de la evaluación del psiquiatra. Como en el estudio original, fueron entrevistados el marido o cónyuge de un paciente casado. En los casos de pacientes solteros, se entrevistó por separado al padre y a la madre. De los 13 pares de familiares se vio a ambos en 10 casos y en los 3 casos restantes sólo se vio a un familiar.

En el estudio original la entrevista con un solo familiar en el momento del ingreso produjo los resultados significativos. Por lo tanto, se consideró esta entrevista como definitiva. Las entrevistas familiares no se re- 
pitieron durante el tiempo de seguimiento ni en el reingreso, ni tampoco se administró al paciente solo. Por razones similares no se celebró la entrevista «conjunta». No obstante, el psiquiatra volvió a aplicar algunas medidas en el transcurso del seguimiento para identificar si se habían producido cambios durante este período. Por ejemplo, se preguntaba al paciente sobre la distribución del tiempo en la familia, si se habían producido alteraciones en la frecuencia del contacto entre él y sus familiares.

Todos, excepto dos, de los 67 pacientes que fueron incluidos en el estudio de seguimiento fueron vistos de nuevo por el psiquiatra. Los dos pacientes restantes, que habían abandonado el área del sudeste de Londres, fueron nuevamente valorados a través de notas del hospital y "de correspondencia personal. Además de repetir el Examen del Estado Actual, el psiquiatra registró cuidadosamente el tratamiento farmacológico durante los meses siguientes al alta, contrastando, cuando era posible, con los registros de pacientes externos. Se siguieron estrictamente los criterios para juzgar si un paciente dado de alta había tomado fenotiacinas o antidepresivos. Si la toma de medicación era discontinua o irregular por espacio de más de un mes durante el período de seguimiento, se consideraba que la persona no había tomado la medicación de forma regular.

\section{TECNICAS DE MEDIDA}

Las técnicas de evaluación conductual psiquiátricas y familiares fueron idénticas a las seguidas en el primer estudio, con una diferencia importante: la abreviación de la entrevista familiar. Las razones para acortar la entrevista pueden encontrarse en Vaughn y Leff (1976). El psicólogo pasó varios meses aprendiendo a utilizar el esquema de la entrevista. Se consiguió una elevada fiabilidad interjueces con los entrevistadores originales valorando cintas del estudio de 1972. El psiquiatra aprendió también la técnica de valoración del número de comentarios críticos. La fiabilidad interjueces entre psicólogo y psiquiatra, en 15 entrevistas, fue 0,86 correlación producto momento.

\section{Valoraciones de la respuesta emocional}

Se obtuvieron valoraciones para todas las escalas utilizadas en el estudio de 1972, incluyendo las tres que constituyen los componentes del índice completo de emoción expresada de los familiares (EE): número de comentarios críticos hechos acerca del paciente, hostilidad e implicación emocional excesiva. En las referencias ya mencionadas pueden encontrarse descripciones detalladas, con ejemplos, de éstas y otras medidas (por ejemplo: demostración de calor, insatisfacción en escalas de cuatro puntos que describen estas áreas de la vida familiar).

\section{Recaída}

Los criterios seguidos para valorar la recaída de esquizofrenia fueron los mismos empleados en el estudio de 1972. Brown et al., distinguieron dos tipos de recaída: el tipo 1 implica un cambio de un estado "normal» o «no esquizofrénico» a un estado de «esquizofrenia», según el procedimiento clasificatorio clínico definido por el CATEGO, clases S o P (Wing, Cooper, Sartorius, 1974); el tipo II incluye una «marcada exacerbación» de síntomas esquizofrénicos persistentes. En el momento de esta nueva eva- 
luación, el psiquiatra no conocía el nivel original de la emoción expresada de los familiares.

La valoración de la recaída fue más difícil en el grupo de depresivos. En cada caso se produjo un período de exención de síntomas depresivos entre el alta y el seguimiento. De los 16 pacientes que recayeron, 14 acusaron síntomas de depresión significativos, valorados de acuerdo con el PSE, durante el seguimiento. Los otros dos pacientes estaban bien cuando se llevó a cabo la entrevista de seguimiento, pero informaron de un episodio depresivo, que persistiọ dos semanas o más, durante los meses entre el alta y el último mes cubierto por el PSE.

\section{RESULTADOS}

\section{Grupo esquizofrénico}

La media de comentarios críticos hechos por los familiares de esquizofrénicos en las dos series no.difiere significativamente $(z=0,173)$ pese a que la entrevista familiar utilizada en nuestro estudio era mucho más breve. La media de observaciones críticas hechas por los 46 familiares en el presente estudio fue de 8,22 (DT $=11,11$ ). Se calculó la media del número de comentarios de la totalidad de los 126 familiares entrevistados en el estudio de 1972, encontrándose que era de 7,86 (DT =14,40). Si se considera que estas dos series de pacientes y familiares están valorados por dos equipos diferentes de investigación y con un intervalo de una década, parece un resultado notable.

En la presente muestra, la media del número de observaciones críticas no difiere significativamente para los dos grupos principales (padres, $x=7,04, D T=7,63$; cónyuges, $x=11,92, D T=17,14 ; t=1,07,36$ g. 1 ., NS).

\section{Indice global de emoción expresada de los familiares (EE)}

Como anteriormente, las escalas individuales fueron, en primer lugar, relacionadas con la recaída. En el estudio de 1972 se utilizó un umbral de 7 comentarios críticos para dividir a las familias en dos subgrupos de emoción expresada, aproximadamente iguales en tamaño. Empleando este mismo punto de corte, e incluyendo familiares que habían mostrado marcada implicación emocional (es decir, unas valoraciones de 4 ó 5 sobre una escala de 5 puntos), en el subgrupo de EE elevada obtuvimos las tasas de recaídas que figuran en la Tabla II(a). Este punto de corte produce una división próxima a la media. Sin embargo, una revisión más estricta de los resultados, reveló que un punto de corte de 6 comentarios críticos produjo una mejor discriminación en términos de proporción de recaídas ( $\mathrm{Ta}$ bla II(b). Teniendo en cuenta la naturaleza arbitraria del punto de corte inicial, nos pareció justificado un ajuste en el nivel de críticas requerido para la asignación al subgrupo de EE alta. Todos los resultados que figuran a continuación están basados en este nuevo punto de corte para la escala de criticismo ${ }^{4}$.

\section{Relación entre el índice de emoción expresada y la recaída}

Los datos correspondientes a. las recaídàs de los 37 pacientes esquizofrénicos, de hogares de alta y baja EE, figuran en la tabla 2(b). Un total de 11 pacientes esquizofrénicos (28 por 100 ) recayó durante el período de 
Relación de la emoción expresada por familiares y recaida

en los nueve meses siguientes al alta

Grupo esquizofrénico

EE de los familiares

No recaen

Recaen

Porcentaje
recaída

a) Punto de corte de criticismo de siete comentarios críticos

Alta

$7+$ comentarios críticos y/o acusada IEE

9

9

50

Baja

1-6: comentarios críticos e IEE no acusada

$$
\mathrm{X}^{2}, \mathrm{p}<0,02
$$

b) Punto de corte de criticismo de seis comentarios críticos

Alta

\begin{tabular}{|c|c|c|c|}
\hline 6: comentarios críticos y/o acusada IEE & 11 & 10 & 48 \\
\hline Baja & . & & \\
\hline 1-5: comentarios críticos e IEE no acusada & 15 & 1 & $\begin{array}{c}6 \\
\text { Test exacto } \\
\text { de Fisher: } \mathrm{p} \\
0,007\end{array}$ \\
\hline
\end{tabular}

seguimiento. De éstos, todos, menos uno, sufrieron recaídas del tipo 1 . Se recuperaron durante algún tiempo después de ser dados de alta; pero presentaron síntomas esquizofrénicos notables al ser readmitidos o durante los nueve meses de seguimiento. Seis pacientes ( 16 por 100 de la muestra total) ingresaron de nuevo en el hospital. Como en el estudio de 1972, existe una asociación significativa entre la EE elevada y la recaída ( $\mathrm{p}$ exacta de Fischer $=0,007$ ).

Es evidente, sin embargo, que la EE de los familiares no es el único determinante de la recaída, ya que más de la mitad de pacientes con familiares de EE alta siguieron bien durante el período de seguimiento. Otros factores (sociales, clínicos, ambientales) deben influir en ese resultado. Se llevaron a cabo diversos análisis para averiguar si la relación entre la EE y la recaída estaba modulada por otros factores. Mencionaremos solamente los más importantes.

\section{Otros factores relacionados con la recaída}

\section{a) Factores clínicos y demogräficos}

Se comparó a los pacientes esquizofrénicos que recayeron con los que se mantuvieron, en términos de los síndromes clínicos incluidos en el PSE que presentaban cuando fueron hospitalizados. Solamente un ítem resultó significativo al nivel del 5 por 100: delirios de grandeza, que fueron más comunes en los pacientes que recayeron. 
Dentro del subgrupo de EE alta se estableció, asimismo, una comparación entre los que recayeron y los que siguieron bien. En este análisis dos items, delirios de grandeza y esquizofrenia residual, se encontraron con más frecuencia en los pacientes que recayeron. Como se hicieron 38 comparaciones, estos hallazgos bien pudieran ser fortuitos.

Ninguna otra variable clínica de las que se midieron ni ninguna otra característica del historial psiquiátrico añadieron nada al valor del índice EE para la predicción de la recaída. Estos resultados concuerdan con los hallazgos del estudio de 1972.

De los muchos factores demográficos considerados, sólo dos se relacionaban con el pronóstico: el sexo y el status matrimonial. La proporción de recaídas en hombres esquizofrénicos dúplica la de mujeres y la proporción de solteros es significativamente mayor que la de casados ( $\mathrm{p}$ exacta $=0,003)$ :

casados: hombres, 25 por 100 ; mujeres, 0 por 100 ; total, 8 por 100 solteros: hombres, 54 por 100; mujeres, 31 por 100; total, 42 por 100

En el estudio de 1972 se encontraron diferencias muy similares en status matrimonial y sexo. Estas diferencias no afectan a la asociación principal entre emoción expresáda y recaída.

\section{b) Deterioro laboral y trastornos de conducta previos}

Siguiendo los criterios de Brown et al. para el deterioro laboral, se consideró que el 43 por 100 de los pacientes esquizofrénicos de la muestra actual presentaba esta característica, dos tercios de ellos pertenecientes a familias con EE alta. La proporción de recaídas en el grupo con un mal ajuste laboral, fue del 37 por 100 y en el grupo de los que no empeoraron tenían un buen ajuste del 24 por 100 (NS). Como en el estudio de 1972, encontramos que el deterioro laboral estaba solamente asociado con la recaída debido a su asociación con el nivel de $\mathrm{EE}$. No recayó ninguno de los pacientes con mal ajuste laboral de familiares con EE baja, mientras que recayeron el 55 por 100 con familiares de EE alta.

Muchas de las evaluaciones de la conducta de los pacientes, antes de ser ingresados, fueron derivados del informe de sus familiares. Se examinó, por diversas vías, su relación tanto con la $\mathrm{EE}$ de la familia como con la recaída. Cuando nos basamos en los mismos criterios seguidos por Brown et al., para valorar la presencia o ausencia de trastornos graves de conducta, obtuvimos casi los mismos resultados. Encontramos, asimismo, que la mayoría de los pacientes esquizofrénicos (62 por 100 en el estudio actual; 75 por 100 en el de 1972) entraban en una de estas dos categorías: bien se les califica «con trastornos» de conducta durante los tres meses anteriores a su hospitalización y viven con familiares con alto nivel de emoción expresada, o bien se les considera «sin trastornos» y viven con familiares de baja emoción expresada. Pero en los casos de los pacientes que no eran congruentes en estos dos factores, el nivel de EE se relaciona con la recaída ( $p$ exacta $=0,027$ ), siendo irrelevante el grado de trastornos conductuales que presentan en los meses que preceden a la hospitalización.

El análisis de Brown et al., no tuvo en cuenta la duración y persistencia de los síntomas o la posibilidad de que algunas conductas podían ser diferencialmente importantes, tanto para la respuestas de los familiares como para el pronóstico. No obstante, al examinar los datos desde distintas perspectivas, se obruvieron resultados similares, indicando que la rela- 
ción entre la EE y la recaída era independiente del trastorno de conducta de los pacientes en los tres meses anteriores a su hospitalización.

\section{c) Factores posteriores al alta}

Se examinaron otros dos factores, que se revelaron importantes en el estudio de 1972: medicación de mantenimiento con fenotiacinas y nivel de contacto directo. Como en el estudio de 1972, ninguno de estos factores revistió significación para el grupo de baja $\mathrm{EE}$, mientras que ambos influyeron en los resultados en el grupo con familiares de elevada EE (Tablas 3 y 4). De hecho, el resultado, por lo que respecta a la terapia de mantenimiento, es estadísticamente significativo $(\mathrm{P}<0,05)$. En el estudio de 1972, una tendencia similar no llegó a alcanzar significación estadística ${ }^{5}$.

TABLA 3

Relación entre la EE de los familiares y toma regular de medicación después del alta y recaida Grupo esquizofrénico

\begin{tabular}{|c|c|c|c|c|c|c|c|}
\hline \multirow[b]{2}{*}{$\begin{array}{l}\text { EE de los } \\
\text { familiares }\end{array}$} & \multicolumn{3}{|c|}{ no medicación } & \multicolumn{3}{|c|}{ medicación } & \multirow{2}{*}{$\underset{\left(\mathrm{X}^{2}\right)}{\stackrel{\bullet}{\text { Significán }}}$} \\
\hline & $\begin{array}{l}\text { No } \\
\text { recaen }\end{array}$ & recaen & $\begin{array}{l}\text { porcentaje } \\
\text { recaída }\end{array}$ & $\begin{array}{l}\text { No } \\
\text { recaen }\end{array}$ & recaen & $\begin{array}{l}\text { porcentaje } \\
\text { recaída }\end{array}$ & \\
\hline Alta & 2 & 7 & 78 & 9 & 3. & 25 & $\begin{array}{c}\text { Test exacto de } \\
\text { Fischer } \\
p=0,024\end{array}$ \\
\hline Baja & 6 & 1 & 14 & 9 & 0 & 0 & NS \\
\hline TOTAL ................ & 8 & 8 & 50 & 18 & 3 & 14 & $p=0,023$ \\
\hline
\end{tabular}

\section{Contribución relativa de varios factores a la recaída}

Habiendo identificado algunos factores individualmente relacionados con la recaída, determinamos su contribución relativa por medio de una matriz de correlaciones. Dicha matriz (tabla 5) muestra que la EE de los familiares está más estrechamente relacionda con la recaída que cualquier otro de los factores considerados, incluida la falta de tratamiento farmacológico preventivo. Cuando el trastorno de conducta (basado en la puntuación total de los síntomas) desapareció parcialmente, no se modificaron los niveles de significación. De hecho, la correlación entre EE y la recaída es, en este caso, mayor $(r=0,52 ; p<0,01)$. Existe evidencia concluyente de que, en los pacientes esquizofrénicos, la relación entre la EE y la recaída se mantiene con independencia de los trastornos de conducta.

\section{Efectos aditivos de factores relacionados con la recaída}

Estos análisis por separado suscitaron nuestro interés en relación con 
TABLA 4

Relación entre la EE de los familiares, tiempo de contacto directo con ellos por semana después de alta y recaida

Grupo esquizof rénico

\begin{tabular}{|c|c|c|c|c|}
\hline & EE de los familiares & No recaen & Recaen & $\begin{array}{l}\text { Porcentaje de } \\
\text { recaídas }\end{array}$ \\
\hline \multicolumn{5}{|c|}{ Tiempo de contacto directo con los familiares (menos de 35 horas) } \\
\hline Alta & 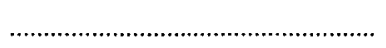 & 5 & 2 & 29 \\
\hline \multirow[t]{3}{*}{ Baja } & (1) & 6 & 1 & 14 \\
\hline & & $\mathrm{X}^{2} \mathrm{NS}$ & & . \\
\hline & EE de los familiares & No recaen & Recaen & $\begin{array}{l}\text { Porcentaje de } \\
\text { recaídas }\end{array}$ \\
\hline
\end{tabular}

Tiempo de contacto directo con los familiares.(más de 35 horas)

\begin{tabular}{|c|c|c|c|}
\hline Alta : & 6 & 8 & 57 \\
\hline Baja . & 9 & 0 & 0 \\
\hline
\end{tabular}

Test exacto de Fischer, $\mathrm{p}=0,006$

TABla 5

matriz de correlación de factores

Grupo esquizofrénico

\begin{tabular}{cccccc}
\hline $\begin{array}{c}1 . \\
\text { Recaida }\end{array}$ & $\begin{array}{c}2 . \\
\text { Trastorno } \\
\text { de conducta }\end{array}$ & $\begin{array}{c}3 . \\
\text { Comentarios } \\
\text { críticos }\end{array}$ & $\begin{array}{c}4 . \\
\text { Medicación }\end{array}$ & $\begin{array}{c}5 . \\
\text { Contacto } \\
\text { directo }\end{array}$ & $\begin{array}{c}6 . \\
\text { Emoción } \\
\text { expresada }\end{array}$ \\
\hline \multirow{2}{*}{1,00} & $-0,20$ & 0,11 & $-0,39 *$ & 0,14 & $0,45 * *$ \\
& & $0,34 *$ & 0,32 & $-0,01$ & 0,24 \\
& & & 0,24 & 0,13 & $0,58 * * *$ \\
& & & & $-0,01$ & 0,01 \\
& & & & & 0,11 \\
\hline
\end{tabular}

Concretamente, este análisis no fue realizado por Brown y sus colegas; pero como los dos estudios eran tan similares en diseño y metodología, fue posible recurrir a los datos originales, reanalizarlos y añadirlos a los nuestros, obteniendo los resultados que se reflejan en la figura 1 .

Resulta evidente, por la proporción de recaídas, que los pacientes con familiares de EE alta, que han pasado mucho tiempo con estos últimos y no han contado con la protección de la medicación de mantenimiento (subgrupo 6), tiene el peor pronóstico. La proporción de recaídas descendió considerablemente cuando opera uno de los dos factores protectores (subgrupos 4 y 5). El mejor pronóstico, sin embargo, lo tienen los pacientes que viven con familiares de EE alta, pero protegidos por un contacto con ellos reducido y por la medicacación de mantenimiento. Para este grupo de pacientes (subgrupo 3), la proporción de recaídas baja al 15 por 100 , proporción significativamente inferior a la de los pacientes sin mecanis- 
Figura 1.-Proporción de recaídas, en nueve meses, de un grupo total de 128 pacientes esquizofrénicos

EE baja $=71$ pacientes $\mathrm{EE}$ alta $=57$ pacientes

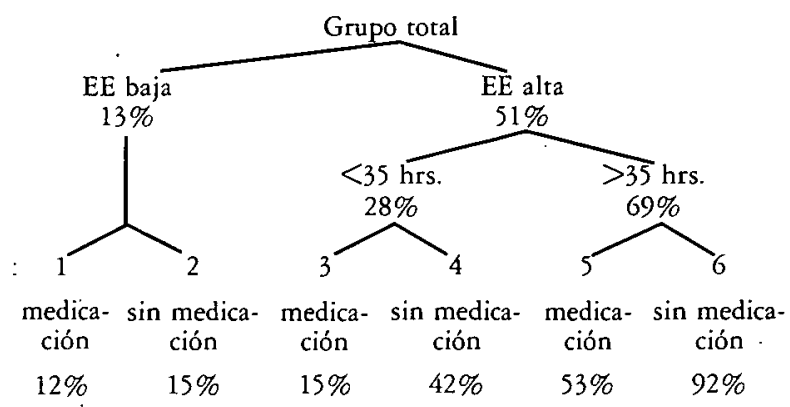

mo protector alguno $(p<0,001)$ y tan reducida como la de los pacientes con familiares de EE baja (subgrupos 1 y 2 ).

Las tasas de recaída en los 6 grupos de la figura 1 proporcionan una valiosa información sobre la función preventiva de la medicación de mantenimiento. De las tasas de recaída en los subgrupos 1 y 2 resulta claro que la medicación no introduce diferencias entre los pacientes que viven con familiares de EE baja. Sí resulta efectiva, no obstante, en la reducción de la proporción de recaídas en pacientes con familiares de EE alta, especialmente en pacientes que pasan menos de 35 horas semanales con sus familiares.

\section{GRUPO DEPRESIVO}

\section{Indice global de emoción expresada (EE) de los familiares}

Si se calcula la media de comentarios críticos, los familiares de los pacientes depresivos no se diferencian de los correspondientes a los grupos de esquizofrénicos $(x=7,19, \mathrm{DT}=9,86)$.

En nuestro intento de aislar las escalas aisladas de emoción expresada con la recaída, utilizamos, en primer lugar, el punto de corte de 7 comentarios críticos, empleado en el estudio de esquizofrenia de 1972, y obtuvimos los resultados reflejados en la tabla $4 a$ ).

Resultó inmediatamente evidente que este umbral no discriminaba adecuadamente entre los pacientes que recayeron y los que se mantuvieron. Otros análisis revelaron que, reduciendo el punto de corte a 2 comentarios críticos, se alcanzó el mejor punto de corte en términos de tasas de recaída

\section{Otros factores relacionados con la recaída}

\section{a) Factores clínicos y demográficos}

Se realizaron análisis de $\mathrm{X}^{2}$ para cada uno de los 38 síndromes incluidos en el PSE, con el fin de determinar si la ausencia, o presencia, de algún síndrome determinado estaba relacionado bien con: a) el pronóstico, a los nueve meses, de pacientes depresivos (recaída/no recaída) dentro del grupo total $(\mathrm{N}=30) ; b)$ pronóstico a los nueve meses, dentro sólo del grupo de elevado criticismo $(\mathrm{N}=21)$; c) si el familiar principal del paciente ex- 
presó elevado criticismo en la entrevista. Sólo uno de los síndromes, irritabilidad, se relacionó con la recaída. En los pacientes que mostraron sentimientos de hostilidad durante el mes anterior al ingreso era más probable la recaída que en los que no habían manifestado ese sentimiento $(p<0,05)$; pero esta relación obedece, casi con seguridad, a la relación entre una valoración positiva de irritabilidad y el criticismo de los familiares $(\mathrm{p}<0,02)$, la variable interviniente que, por sí misma, predice la recaída.

Considerando por separado el grupo de elevado criticismo, no se encontraron diferencias respecto a la presencia/ausencia de síndromes del PSE entre los pacientes que recayeron y los que se mantuvieron a lo largo del período de seguimiento.

Otras variables, como características de la historia psiquiátrica del paciente, no tuvieron, en general, relación con el pronóstico en el grupo de depresivos. Así, por ejemplo, el sexo del paciente, el estado al ingreso y el tiempo transcurrido desde el primer episodio, no fueron significativos. La influencia del estado civil no pudo examinarse ya que sólo 2 , de los 30 pacientes depresivos, no estaban casados y viviendo con sus esposas cuando fueron ingresados. Se aislaron, sin embargo, dos factores que interactuaron con el criticismo de los familiares y el pronóstico: la edad al ser ingresados y la edad cuando padecieron el primer episodio. Los 5 pacientes que tenían 45 años o más cuando fueron ingresados y que volvieron con sus familias de elevado criticismo, fueron extremadamente vulnerables: todos ellos recayeron. Este porcentaje es significativamente más elevado que el 20 por 100 de recaídas de pacientes del mismo grupo de edad, pero que vivían con familiares de bajo criticismo ( $\mathrm{p}$ exacta $=0,01$ ).

- El criticismo de los familiares tuvo una influencia menor en el pronóstico de los pacientes que tenían menos de 45 años al ser ingresados. Este resultado no alcanzó significación estadística, si bien los hallazgos van en la misma dirección.

La edad en que sufrieron el primer episodio juega también un papel significativo en la determinación de qué pacientes deprimidos se mantèndrán bien. Los pacientes cuyos primeros episodios depresivos ocurrieron cuando eran muy jóvenes $(<.21$ años) o de media edad $(>45$ años), mostraron una probabilidad significativamente mayor de recaída que aquellos cuyo primer episodio se produjo entre los 21 y 45 años ( $p$ exacta=0,001). Dentro. del grupo con elevado criticismo, esta relación se mantiene $(\mathrm{p}=<0,004)$. Pero, nuevamente, la presencia de un bajo criticismo familiar ejerce una influencia protectora y cuando se controla la edad en el primer episodio, subsiste la principal relación entre criticismo y pronóstico ( $\mathrm{p}$ exacta $=0,018$ ).

\section{b) Deterioro laboral y trastorno de conducta previos}

Entre los pacientes depresivos era poco frecuente el deterioro laboral, tal como éste fue definido por Brown et al., (1972): sólo dos presentaron (tabla 4b). Decidimos que todo paciente depresivo, cuyo familiar principal hubiera hecho dos o más comentarios críticos, fuera asignado al subgrupo de elevado «criticismo». Todos los resultados que figuran a continuación están basado en este.umbral de "criticismo».

Los otros dos índices de EE alta (hostilidad e implicación emocional excesiva) nada añaden a la asociación significativa entre comentarios críticos y recaída en el grupo depresivo. El índice para este grupo de pacientes decidimos denominarlo índice de «criticismo» para distinguirlo del índice, diferentemente establecido, de emoción expresada (EE) empleado para los dos grupos de pacientes esquizofrénicos. 
Relación del criticismo de los familiares y recaida en los nueve meses después del alta Grupo depresivo

\begin{tabular}{lccc}
\hline & No recaen & Recaen & $\begin{array}{c}\text { Porcentaje } \\
\text { recaídas }\end{array}$ \\
\hline
\end{tabular}

a) Punto de corte de criticismo de siete comentarios críticos

$\begin{array}{lrrr}7+\text { comentarios críticos .......................... } & 4 & 6 & 60 \\ 0-6: \text { comentarios críticos ....................... } & 10 & 10 & 50\end{array}$

\section{$\mathrm{X}^{2} \mathrm{NS}$}

b) Punto de corte de criticismo de dos comentarios críticos

$2+$ comentarios críticos

$2<$ comentarios críticos

$\begin{array}{rr}7 & 14 \\ 7 & 2\end{array}$

Test exacto de Fisher $\mathrm{p}=0,032$

\section{Relación entre el índice de emoción expresada y la recaída}

Los pacientes depresivos de nuestra muestra fueron, incluso, más vulnerables a los efectos de las críticas de los familiares que los ésquizofrénicos y tienden a recaer con un nivel más bajo de criticismo. En la tabla 4 b) figuran los datos de recaídas de 30 pacientes con familiares de alto y bajo criticismo. Dieciséis, de los 30 (53 por 100 del grupo total) recayeron durante el período de seguimiento. Siete, de los. 16 pacientes que recayeron (23 por 100 del grupo total), fueron reingresados en el hospital. Los pacientes cuyos familiares expresaron dos o más comentarios críticos (en la entrevista principal) recayeron en proporción significativamente más elevada que los pacientes con familiares menos críticos (test exacto de Fischer, $\mathrm{p}=0,032)$.

un déficit serio, por espacio de tres meses, en los dos años precedentes a la hospitalización. Entre los pacientes depresivos y los esquizofrénicos existen diferencias significativas en las proporciones totales de: pacientes con deterioro laboral ( 6 por 100 vs. 43 por $100, p<0,01$ ); pacientes varones sin empleo al ser ingresados ( 0 por 100 vs. 40 por 100 , p exacta $=0,022$ ); mujeres que presentan marcado deterioro en las tareas domésticas ( 5 por 100 vs. 45 por 100 , p exacta $=0,003$ ). En todos los casos el mayor deterioro se da en el grupo esquizofrénico.

En cuanto al rango y severidad de los síntomas mencionados, los trastornos de conducta, en los meses anteriores a la hospitalización, fue similar en los dos grupos clínicos; pero en los pacientes deprimidos existía una relación más estrecha entre el trastorno (valoración total de síntomas) y el número de observaciones críticas hechas por los familiàres sobre los pacientes (grupo esquizofrénico: $r=0,33, p<0,05$; grupo depresivo: $r=0,48, p<0,01)$. La cantidad de criticismo se relaciona, a su vez, con la 
duración de la entrevista (Vaughn y Leff, 1976), de donde resulta un modelo típico de respuesta de los familiares de pacientes depresivos: cuanto más hablan los familiares, más probable es un mayor criticismo y más elevada la proporción de trastorno del paciente. Sin embargo, la matriz de correlación revela que el trasstorno de conducta no está relacionado con el pronóstico a los nueve meses (tabla 7).

TABLA 7

Matriz de correlación de factores

Grupo depresivo

\begin{tabular}{|c|c|c|c|c|c|}
\hline $\begin{array}{c}1 . \\
\text { Recaida }\end{array}$ & $\begin{array}{c}2 . \\
\text { Trastorno } \\
\text { de conducta }\end{array}$ & $\begin{array}{c}3 . \\
\text { Comentarios } \\
\text { críticos }\end{array}$ & $\begin{array}{c}4 . \\
\text { Medicación }\end{array}$ & $\begin{array}{c}5 . \\
\text { Contacto } \\
\text { directo }\end{array}$ & $\begin{array}{c}6 . \\
\text { Emoción } \\
\text { expresada }\end{array}$ \\
\hline \multirow[t]{2}{*}{1,00} & 0,15 & $\begin{array}{c}0 \\
0,49 * *\end{array}$ & $\begin{array}{r}0,13 \\
-0,11 \\
-0,31\end{array}$ & $\begin{array}{c}0,04 \\
-0,20 \\
-0,45^{*} \\
0,30\end{array}$ & $\begin{array}{c}0,41^{*} \\
0,38^{*} \\
0,49^{* *} \\
-0,22 \\
-0,40^{*}\end{array}$ \\
\hline & * $\mathrm{p}$ & & $* *$ & $(0,01$ & \\
\hline
\end{tabular}

Factores posteriores al alta

Solamente una pequeña proporción de pacientes depresivos (20 por $100, N=6$ ) estaba bajo medicación preventiva en el momento del seguimiento, número excesivamente exiguo para deducir conclusiones acerca de los efectos de esta variable sobre el pronóstico. La frecuencia del contacto directo entre pacientes y familiares no mantenía relación con los modelos de recaída. Pero los pacientes depresivos, cuyos familiares habían expresado dos o más observaciones críticas, tenían significativamente menos contacto con los miembros de su familia que los pacientes con familiares con bajo criticismo ( $\mathrm{p}$ exacta $=0,024)$.

\section{Contribución relativa de varios factores a la recaída}

La matriz de correlaciones (tabla 7) muestra que el criticismo de los familiares es el único, entre los factores considerados, que contribuye de manera significativa a la recaída $(p<0,05)$. Cuando el trastorno de conducta se excluye, en un análisis.de regresión múltiple, existe todavía una correlación significativa entre criticismo y recaída. De hecho, si se controla el criticismo, la contribución de los demás factores de la matriz a la recaída es insignificante.

\section{DISCUSION}

Los principales resultados de Brown et al., (1972), en relación con los factores familiares y sociales que influyen en el curso de la esquizofrenia, 
han sido replicados casi exactamente. Ha sido posible predecir patrones de recaída de modo incluso más preciso que en el estudio anterior, considerando los efectos añadidos de varios factores biológicos y sociales, que se han demostrado importantes aisladamente en relación con el pronóstico. Un nivel elevado de emoción expresada de los familiares, en el momento del ingreso del paciente, sigue siendo el mejor predictor aislado de recaída sintomática durante los nueve meses siguientes al alta. Pero resulta sugerente que la combinación de la. medicación de mantenimiento y la reducción del contacto directo con un familiar con un alto nivel de implicación emocional o de criticismo puede evitar la recaída en casi todos los casos.

\section{Diferencias de sexo y del grupo familiar}

Brown et al., creían que las diferencias en la proporción de recaídas de esquizofrénicos solteros y casados eran consecuencia de que si uno de los padres, o ambos, vivía(n) con el paciente solo, existía un mayor nivel de emoción expresada. Pero éste no ha sido el caso en nuestro estudio; había la misma probabilidad de que los padres presentaran EE alta aunque en la familia conviviesen otros miembros. Tampoco pueden explicarse los resultados por diferencias en el nivel de emoción expresada por los padres y cónyuges, ya que éstas no eran significativas.

Los datos, tanto del estudio de 1972 como del nuestro, muestran que los varones solteros presentan un mayor riesgo de recaída y que làs mujeres casadas son menos vulnerables. Ambos estudios presentan evidencia de que los varones solteros de hogares con EE alta están significativamente menos protegidos por la medicación y el reducido contacto directo que las mujeres en condiciones semejantes ( $p$ exacta $=0,006$ ). Estos mecanismos protectores sólo se detectaron en el 5 por 100 de estos hombres, frente al 50 por 100 de las mujeres solteras.

Sólamente caben especulaciones sobre las razones de la baja proporción`de recaídas en las mujeres esquizofrénicas casadas: pueden concurrir factores como la personalidad premórbida o diferentes expectativas de la función a desempeñar. No es éste el lugar para extendernos sobre este hallazgo. La importancia de estos resultados reside en la identificación de un grupo que sería considerado de alto riesgo de recaída en cualquier programa preventivo.

\section{Factores posteriores al alta}

Comparados con nuestras muestras de pacientes esquizofrénicos, en el estudio de 1972 recayó una mayor proporción de pacientes mientras tomaban medicación. Estas diferentes tasas de recaída pueden deberse a los criterios más restrictivos utilizados en este estudio en cuanto al concepro de «medicación regular». Por otra parte, habían transcurrido diez años desde que se realizó el primer estudio. Es posible qué actualmente se observe una mayor discriminación en cuanto a la prescripción y administración de drogas a pacientes que, con frecuencia, tienen una mayor necesidad.

Un reducido contacto directo puede deberse a que el paciente salga de casa, por ejemplo, a trabajar o a un Centro de Día o puede producirse permaneciendo en casa por aislamiento social. Es necesario distinguir ambos términos: "aislamiento social» se refiere a una disminución en la comunicación verbal, como rechazo a iniciar una conversación, no responder cuan- 
do se le pregunta directamente, apariencia de que vive en otro mundo. El contacto directo es, realmente, una medida de proximidad física. Así, es posible para una persona tener un elevado contacto directo con sus familiares y seguir con un elevado aislamiento social.

Brown y otros sugirieron que el aislamiento social puede ser un medio de afrontar una situación de tensión, un mecanismo protector que disminuye las posibilidades de recaída en la esquizofrenia. La investigación de esta posibilidad reveló una asociación significativa entre contacto directo reducido y aislamiento social en pacientes con familiares de alta $\mathrm{EE}$ ( $\mathrm{p}$ exacta $=0,023)$. En el grupo de alta EE, dos tercios de los sujetos socialmente aislados, o que evitaban a los miembros de la familia durante los meses anteriores a su primera hospitalización, se mantuvieron asintomáticos durante el seguimiento, en tanto que el 58 por 100 de los que no presentaron señales de aislamiento, recayeron más tarde. Ello sugiere un estilo general de enfrentamiento y apoya la noción de que la persona que sufre esquizofrenia ejerce algún control sobre el curso de su enfermedad.

\section{Comparación entre las pautas de recaída en esquizofrénicos y depresivos}

Uno de los objetivos del presente estudio era determinar si los factores que influían en el diagnóstico eran, de algún modo, específicos de la esquizofrenia, para lo que se incluyó una muestra de pacientes depresivos neuróticos. Nuestros resultados sugieren que el modelo de recaída de los dos grupos clínicos es diferente. En la muestra estudiada existe una relación significativa entre el criticismo de los familiares y la recaída, sugiriendo que los pacientes psiquiátricos no esquizofrénicos se ven también afectados por la calidad de sus relaciones emocionales con sus familiares. No obstante, los pacientes depresivos resultan más sensibles al criticismo que los pacientes esquizofrénicos. Por otra parte, los mecanismos protectores, tan importantes en el pronóstico de pacientes esquizofrénicos con familiares de EE alta (medicación de mantenimiento y contacto reducido con los familiares) no tienen relación con las pautas de recaída del grupo depresivo.

Además, parece haber algo específico en cuanto a las formas de respuesta de los esquizofrénicos a su entorno social. Por ejemplo, un paciente confrontado con un familiar de EE alta puede reaccionar bien aislándose o evitándole, reduciendo el contacto directo como consecuencia de esta maniobra protectora. Esto constituye una pauta común entre los pacientes esquizofrénicos. Sin embargo, cuando se intenta explicar la relación entre el contacto directo reducido y el elevado criticismo en el grupo de sujetos de.presivos, la evidencia contradice esta interpretación. Aquí es más probable que exista una relación pobre entre el paciente y el familiar que anteceda al trastorno, y se caracteriza por un contacto directo reducido y una comunicación pobre. Cuando el episodio se inicia, el familiar responde con críticas e informa de un trastorno de conducta muy importante (que puede ser, o no, exacto). En cualquiè caso, es la respuesta del familiar, más que la gravedad de la enfermedad, el mejor predictor de la recaída del paciente dentro de un período de tiempo determinado.

\section{Aplicaciones prácticas}

El presente artículo enfatiza la importancia de la EE de los familiares sobre el pronóstico, inicialmente señalada por Brown y sus colegas. Sin embargo, queda claro que los efectos negativos de la EE alta de los familiares 
pueden modificarse mediante dos mecanismos protectores: medicación y reducido contacto directo (fig. 1). Una de las mayores dificultades en los intentos para tratar a las personas esquizofrénicas ha sido la falta de conocimiento de los objetivos a alcanzar a través de la intervención. Nuestros datos sugieren que si se pudiera intervenir para asegurar que los pacientes con familiares de alta EE, que constituyen un grupo de alto riesgo, tomaran de forma regular la medicación y tuviesen el mínimo contacto con sus familiares, es muy posible que se redujera considerablemente su tasa de recaídas, hasta alcanzar la de pacientes con familias de EE baja.

Del resumen de factores relacionados con la recaída se desprende directamente qué sujetos presentarían el riesgo más elevado en cualquier programa preventivo. Para quedar mínimamente protegido contra la recaída, un soltero que vive con familiares de EE alta debe, por una parte, tomar medicación regularmente y tener el menor contacto posible con sus familiares. Para las mujeres solteras y los varones casados, bastaría con la presencia de uno de los dos factores protectores, en tanto que las mujeres casadas podrían, presumiblemente, mantenerse bien incluso sin medicación y con un mayor contacto con sus familiares.

El primer paso, en todo programa de intervención, debería ser identificar las familias en las cuales el paciente esquizofrénico puede presentar un alto riesgo de recaída. Aunque la entrevista familiar de Brown y Rutter se ha utilizado, hasta el presente, sólo como instrumento de investigación, es muy posible que una versión simplificada, como la desarrollada por Vaughn y Leff (1976), pudiera adoptarse como instrumento clínico práctico. Pero la importancia de un instrumento estandarizado, administrado por un entrevistador debidamente entrenado, debe ser enfatizada.

Sería, evidentemente, también una ventaja poder identificar pacientes de alto riesgo en términos de algunas medidas psicofisiológicas de estrés. En los esquizofrénicos crónicos se han apreciado niveles de activación sorprendentemente elevados (Venables, 1960). Tal vez fuera posible detectar anormalidades en las respuestas psicofisiológicas de estos pacientes e identificar, por esta vía, aquellos que son más sensibles a su entorno social.

Una vez identificados los pacientes de alto riesgo, hay algunas posibilidades de intervención. La medicación, de mantenimiento es, quizá, el factor protector aislado más importante para pacientes con familiares de EE alta. Pero la recaída de los pacientes de más alto riesgo no podrá evitarse con medicación únicamente. Es necesario encontrar medios de -reducir el contacto directo entre el paciente y sus familiares con EE alta. La solución obvia, para las personas que viven con sus padres, es llevarlas a un centro institucional u otro alojamiento protegido, pero esto no siempre es posible. La estrategia alternativa sería que el paciente tuviese un trabajo diurno, lo que produciría en él una sensación de independencia y reduciría el número de horas de convivencia entre paciente y familia.

En última instancia, no obstante, no es probable que estas soluciones «administrativas» sean efectivas sin la cooperación del paciente y de sus familiares. Los subgrupos de la figura 1 no están constituidos al azar sino por autoselección. Puede haber tipos de relación que mantienen unida a la familia pese a existir una tensión considerable. En tales casos, probablemente los familiares se resistirán a intentos externos de llevar al paciente a otro lugar, a pesar de que esta sugerencia pueda hacerse con mucho tacto, lo que se produce, especialmente, en familias cuyos miembros presentan una implicación emocional excesiva. Si no se conocen estas dificultades potenciales y no se abordan previamente en un programa de intervención, éste puede estar condenado al fracaso. 
Esto nos lleva a otro tipo de intervención: esforzarnos en cambiar las actitudes de los familiares con una implicación emocional excesiva o altamente críticos. Hasta ahora conocemos muy poco sobre la formación de tales actitudes o de su susceptibilidad de cambio, lo que convierțe esta sugerencia en muy especulativa. Pero un análisis de las determinantes de la emoción expresada de los familiares puede darnos idea de cuál puede ser la mejor estrategia de intervención.

Por ejemplo, al considerar sobre cómo tratar con un familiar altamente crítico, sería útil examinar, sistemáticamente, las quejas del familiar para realizar, en efecto, un análisis del contenido de sus respuestas. ¿Se dirigen sus observaciones a los rasgos de personalidad persistentes en el paciente o se refieren, principalmente, a las conductas relacionadas con la enfermedad? Actualmente estamos llevando a cabo este trabajo.

Han transcurrido quince años desde que Brown y sus colegas empezaron a explorar la complejidad de las relaciones entre el esquizofrénico y su entorno social. El conocimiento actual sobre los factores que influyen en el pronóstico es tal que, finalmente, podemos empezar a planificar programas eficientes para prevenir la recaída en la esquizofrenia. Posiblemente las nuevas técnicas de terapia conductual pueden proporcionar instrumentos para una intervención racional. A pesar de la magnitud del proyecto, puede que no haya una ocasión mejor que la presente.

\section{Notas}

1 Publicado originalmente en el British Journal of Psychiatry, 1976, 129, 125-137.

${ }^{2}$ El número de críticas y la hostilidad se basaban sea en la emoción negativa (juzgada por el tono de la voz) o una «clara expresión de resentimiento, desaprobación, disgusto o rechazo». La implicación emocional excesiva se daba en los padres más que en otros familiares y se caracterizaba por ansiedad excesiva, preocupación o protección exageradas hacia el paciente. Se valora en base a los sentimientos expresados en la entrevista o la conducta observada fuera de ella.

3 Todos éstos eran casos en que el paciente tenía una larga historia psiquiátrica y los familiares, generalmente muy críticos para con el paciente, no deseaban repetir otra vez toda la historia. Una respuesta típica fue: «Para mí es una experiencia terrible y una pérdida de tiempo». Todos los paicentes esquizofrénicos excluidos vivían con sus padres.

4 En ningún caso se registró hostilidad en ausencia de un elevado nivel de criticismo, por lo que esta escala no se utilizó en la formación del grupo de EE alta.

5 El 75 por 100 de los pacientes esquizofrénicos se ajustaron a nuestros criterios de toma regular de la medicación. Proporciones similares. de pacientes de baja y alta EE no tomaron regularmente la medicación.

\section{Referencias}

Brown, G. W.; BiRLEY, J. L. T., y WING, J. K. «Influence of family life on the course of schizophrenic disorders: a replication». Britsh Hournal of Psychiatry, 1972, 121, 241-258.

BROWN, G. W., y RUTTER, M. «The measurement of family activities and relationships: a methodological study». Human Relations, 1966, 19, 241-263.

RUTTER, M., y BRown, G. W. "The reliability and validity of measures of family life and relationships in families containing a psychiatric patient». Social Psychiatry, 1966, 1, 38-53.

VAUGHN, C. E., y LEFF, J. P. «The measurement of expressed emotion in the families of psychiatric patients. Accepted for publication». British Journal of Social and Clinical Psychology, 1976.

VENABLES, P. H. «The effect of auditory and visual stimulation on the potential response of schizophrenics». Brain, 1960, 83, 77-92.

WING, J. K.; COOPER, J. E., y SARTORIUS, N. The Description and Calssification of Psychiatric Symptoms: An . Introduction Manual for the PSE and Catego System. Londres: Cambridge Press, 1974. 